\title{
Pelvic reconstruction with bone cement and total hip prosthesis after resection of chondrosarcoma. Case report
}

\author{
Ioan Mihai Japie*, Adrian Bădilă***, Radu Rădulescu***, Eduard Mitroi*, Andra Bujdei*, \\ Adrian Dumitru*****, Răzvan Ene***, Alexandru Papuc*, Cătălin Cîrstoiu* ** \\ *Department of Orthopaedics and Traumatology, Emergency University Hospital Bucharest \\ ** "Carol Davila" University of Medicine and Pharmacy, Bucharest \\ ***Department of Pathology, Emergency University Hospital Bucharest
}

Correspondence to: Bădilă Adrian, Lecturer, $\mathrm{MD}, \mathrm{PhD}$, Department of Orthopaedics and Traumatology, Emergency University Hospital Bucharest 169 Splaiul Independentei Street, Code 050098, Bucharest, Romania, Phone: +4021 31805 23, E-mail: adrian_emilian_badila@yahoo.com

\begin{abstract}
Introduction. Chondrosarcoma is a malignant tumor of cartilaginous origin representing approximately $20-30 \%$ of all bone malignant tumors and occupying the second place in terms of their incidence. It usually affects adults between 40 and 60 years old, but can be encountered at older ages as well.

Materials and methods. We report the case of a 55 -year-old male patient who presented to our department with 2 weeks-long right hip pain and partial loss of functionality in right limb. We performed X-rays, magnetic resonance imaging, computed tomography, bone scintigraphy, and angiography, which established the diagnosis of pelvic tumor. Biopsy of the tumor was performed by iliofemoral approach and its result showed moderately differentiated chondrosarcoma. Orthopaedic surgery was performed, with tumoral removal within oncological limits, pelvic reconstruction using acrylic bone cement impregnated with Vancomycin and total hip arthroplasty.

Results were assessed using the Musculoskeletal Tumor Rating Scale and a score of 23 out of 35 was obtained (the higher the better). Postoperative complications consisted in flap-skin necrosis that resolved within 2 months after surgery.

Conclusion. Bone defects repair using antibiotic impregnated cement drastically reduced the rate of postoperative infections, thus decreasing both morbidity and mortality. In spite of technological advancement, long-term prognosis remains reserved in chondrosarcoma, due to its specific aggressivity, resistance to chemo- and radiotherapy and high rate of recurrence.

Keywords: chondrosarcoma, radiotherapy resistant tumor, bone tumor, hip arthroplasty, bone cement
\end{abstract}

\section{Introduction}

Chondrosarcoma is a malignant tumor of cartilaginous origin representing approximately $20-30 \%$ of all bone malignant tumors and occupying the second place in terms of their incidence. The tumor is primary in $90 \%$ of the cases, but it can also be secondary to malignant degeneration in preexisting conditions such as Ollier disease or Maffucci syndrome [1]. It usually affects adults between 40 and 60 years old, but can be encountered at 
older ages as well [2].

The most common areas, in which chondrosarcoma occurs are the proximal femur and pelvis, especially the ilium and the area surrounding the acetabulum. Other affected areas, in decreasing frequency, are the proximal humerus, the scapula and the distal femur [3]. Chondrosarcoma is a chemo- and radiotherapy resistant tumor, which makes surgical treatment the only viable option. The purpose of orthopaedic surgery is that of resecting the tumor within oncological safety limits, achieving good limb functionality, as well as maintaining or increasing the quality of life, which are all more difficult to achieve in pelvic tumors due to the anatomical and biomechanical features of the area.

\section{Materials and methods}

We present the case of a 55-year-old male patient who presented to our department with 2 weeks-long right hip pain and partial loss of functionality in right limb. The symptoms occurred spontaneously, without any history of injury or trauma.

Clinical examination revealed antalgic loss of articular mobility in the right hip and tenderness at palpation, but without any discernable mass. The anteroposterior view $\mathrm{X}$-rays showed a tumoral mass of the right hip involving the acetabulum and right iliac wing. Advanced investigations like magnetic resonance imaging (MRI), computed tomography (CT), bone scintigraphy, and angiography were performed afterwards.

The MRI revealed a bone tumor involving the acetabulum with right iliac wing extension and intimate contact with the adjacent muscular structures (Fig. 1).

The CT scan (Fig. 2) showed a polilobular bone tumor which penetrated the cortical bone and expanded to the internal obturator muscle.

The immediate phase of the scintigraphic exam revealed discrete hypercaptation in the soft tissue surrounding the right coxofemoral joint, while in the metabolic phase (Fig. 3), a moderate heterogeneous hypercaptation was revealed at the acetabular site, denouncing a metabolic active process.

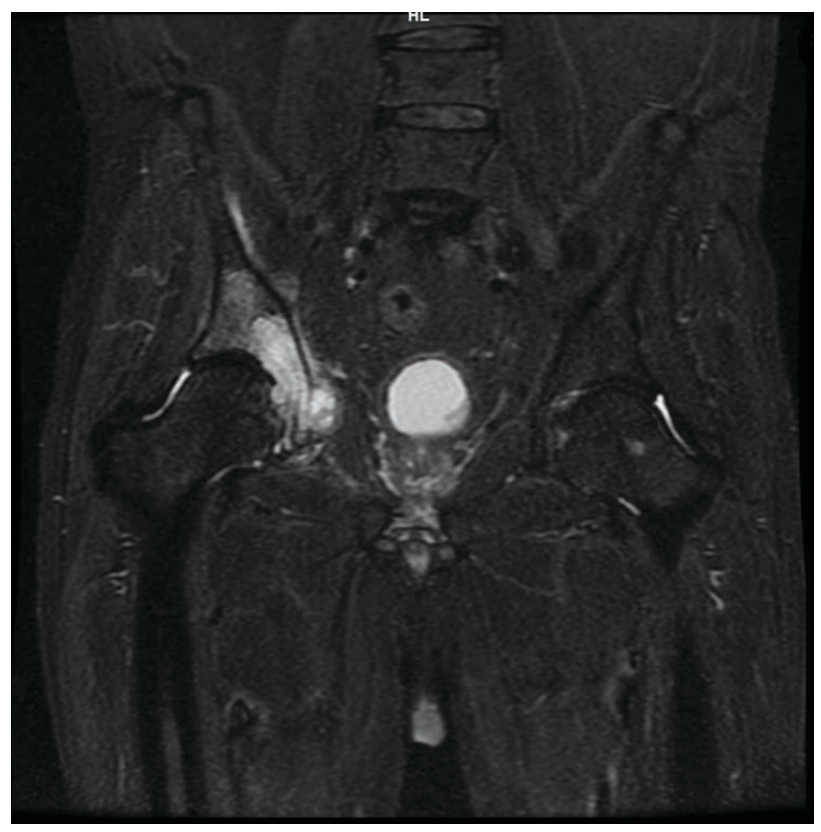

Fig. 1 MRI aspect showing bone tumor involving the acetabulum with right iliac wing extension

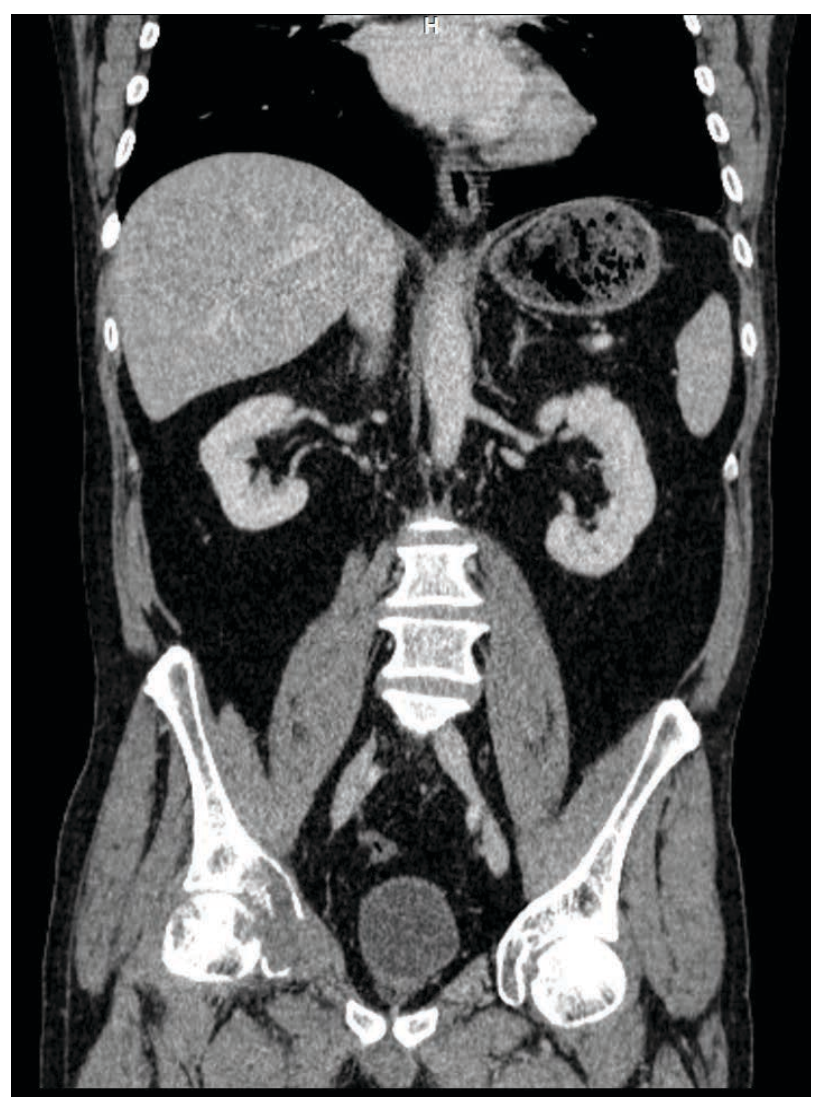

Fig. 2 The bone tumor penetrates the cortical bone and expands to the internal obturator muscle 


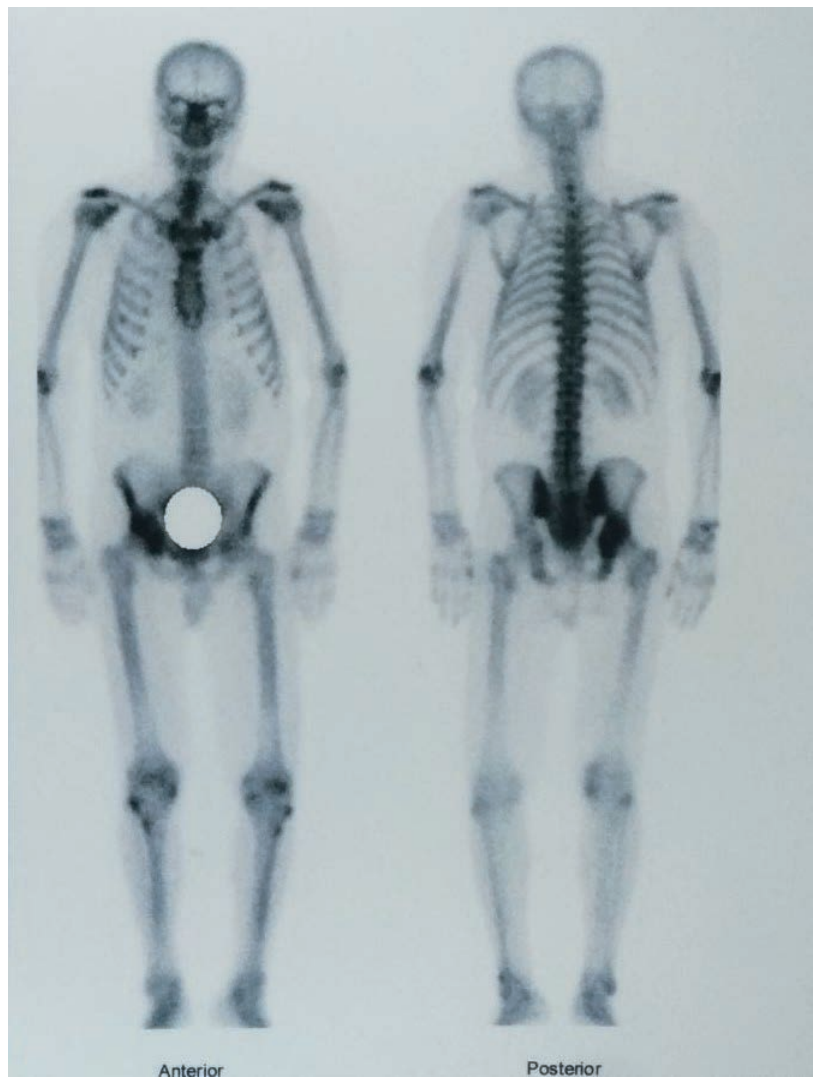

Fig. 3 Moderate heterogeneous hypercaptation at the acetabular site

The angiography completed the imaging investigations, showing a slight loading with "tumoral blush" aspect in the projection area of the lesion, originating from the circumflex iliac artery. Notably, the internal margin of the common femoral artery had a slight irregular aspect, probably due to tumoral infiltration of the area.

Biopsy of the tumor was performed by iliofemoral approach and its result showed moderately differentiated chondrosarcoma (grade G2) (Fig. 4,5).

Orthopaedic surgery was performed, using both iliofemoral and Kocher-Langenbeck approaches, with tumoral removal within oncological limits, pelvic reconstruction using acrylic bone cement impregnated with Vancomycin, reinforced by two Schanz pins and one iliopubic plate with screws. The joint reconstruction ensued, using total hip cemented prosthesis (Fig. 6).

The histological examination of the resected tumor revealed a poorly differentiated chondrosarcoma (grade $\mathrm{G}_{3}$ ) without invasion of the resection margins (Fig. 7).

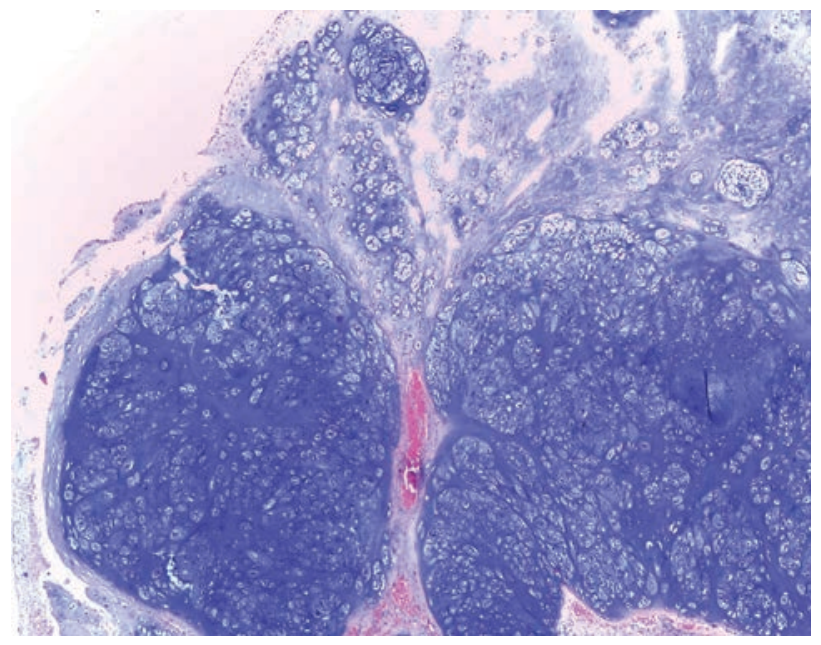

Fig. 4 Grade 2 chondrosarcoma with a multilobulated appearance. The lobules are separated by fibro-vascular bands. The chondrocytes, vary in size and shape, and contain enlarged hyperchromatic nuclei. Binucleation is frequently seen (H.E. ob. 100X)

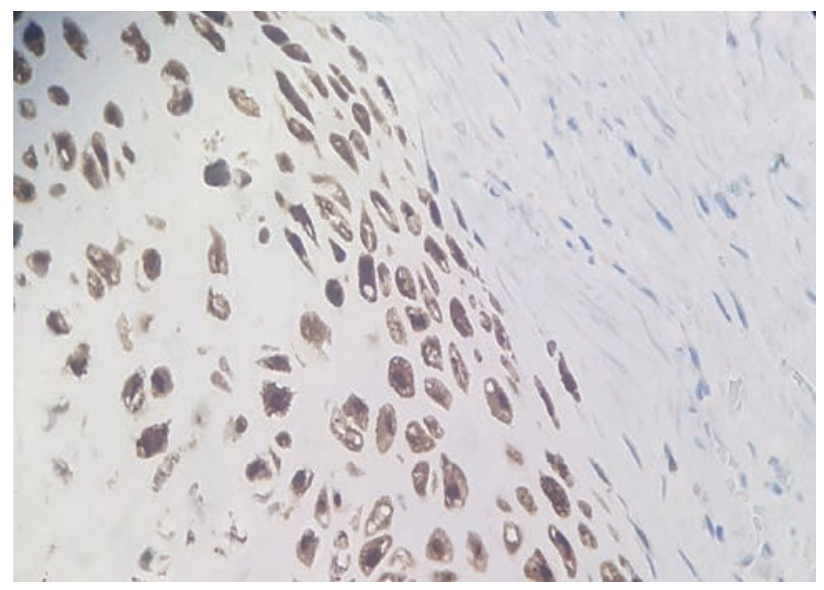

Fig. 5 Grade 2 chondrosarcoma showing specific, intense nuclear and cytoplasmic positive immunostaining for S100 of the atypical chondrocyte (IHC stain with DAB chromogen, ob. 200x)

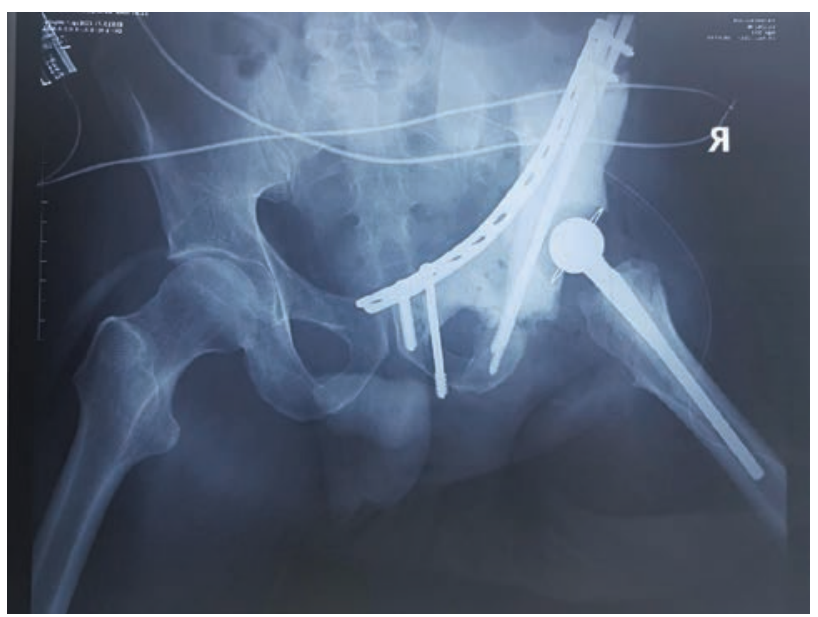

Fig. 6 Postoperative anteroposterior view $X$-ray 


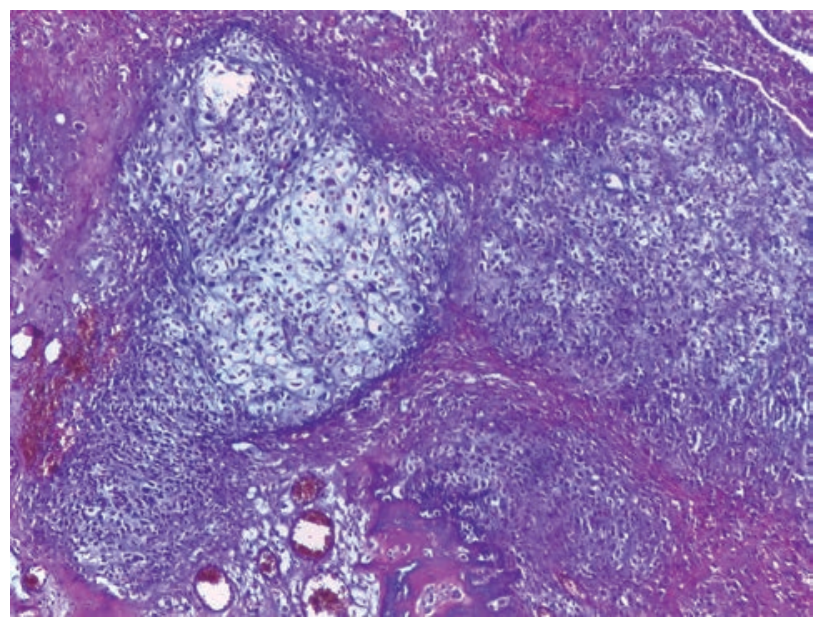

Fig. 7 Grade 3 chondrosarcoma with marked hypercellularity, extreme pleomorphism with markedly hyperchromatic nuclei; bizarre tumor giant cells and small cells, frequent mitotic figures. Focal hemorrhagic areas and exuberant neovascularization (H.E. ob. 200x)

\section{Results}

Results were assessed using the Musculoskeletal Tumor Rating Scale (MSTS) that evaluates the range of motion, pain, stability, deformity, strength (in abduction of hip), function activity, and emotional acceptance. A score of 23 out of 35 was obtained (higher is better).

Postoperative complications consisted in flap-skin necrosis that resolved within 2 months after surgery.

\section{Discussions}

The histopathological classification of chondrosarcomas is useful both from a therapeutical point of view and as a predictive factor. Considering cellularity, cellular atypia, and mitosis degree, chondrosarcomas can be classified into 3 types:

1st degree - moderate cellularity, hyperchromatic uniform nuclei;

2nd degree - the presence of cellular atypia and mitoses (mitotic activity);

3rd degree - nuclear pleomorphism and abundant mitotic activity.

The histopathological degree is regarded as inversely proportional with the patient's survival rate in literature, i.e. the 10-year survival rate decreases from 100\% [4] in chondrosarcoma of the 1st degree to $90 \%$ for 2 nd degree and merely $55 \%$ for the 3 rd degree $[5,6]$.

Another important prediction factor is the location of the tumor and the adopted type of surgical procedure. At first Enneking and Dunham [7] and then Kollender [8] described 5 types of osteotomy in the pelvic region: the iliac, acetabular, ischiopubic, sacroiliac and type $\mathrm{H}$ if the femoral head is affected. Of all these, acetabular hemipelvectomy, alone or combined with any other type, achieved the worst results and the most frequent complications, probably because of the loss of an important support segment of the coxofemoral joint. Thus, Puri's study in 2013 highlights the high complexity of hemipelvectomy involving the acetabular area by increasing the duration of surgery from an average of 4.5 hours to 6.8 hours and also a significantly higher intraoperative bleeding in acetabular lesions with an average of $4500 \mathrm{ml}$ versus $2500 \mathrm{ml}$. Postoperative results were quantified using the MSTS score, which was lower in patients requiring type 2 hemipelvectomy (with a median score of 22 versus 27) [9].

Tumor surgery in the pelvis is complicated both from a technical point of view, due to the vasculonervous and visceral structures, and due to the complications that may occur (20-60\% of the cases) $[10,11]$ - infections, pelvic limb inequality, skin necrosis, prosthesis dislocation, lesions involving the femoral vessels or the sciatic nerve $[12,13]$. Of all these, the most frequent appeared to be the occurrence of postoperative infection, possibly due to the increased duration of surgery, large bone defects after tumor excision, implantation of synthetic materials, proximity to the rectum and the genitourinary tract [14]. According to Buchholz's theory, as the local antibiotic levels are 200 times higher in the case of antibiotic-impregnated cement than by systemic administration, the infection rate can be diminished by using antibiotic- 
impregnated cement $[15,16]$. Also, a Donati study concludes that the use of a cement spacer impregnated with Vancomycin diminishes the rate of infection in reconstruction resections techniques used for bone tumors of the pelvis [17].

The poor long-term prognosis of chondrosarcomas is also a result of increased incidence of recurrences and metastases. In literature, recurrences appeared in $15-35 \%$ of the cases and metastases in $20-40 \%[9,12,18]$. The high rate of recurrence can be explained by the lack of adjuvant treatment, inappropriate surgical approach, and surgical reintervention [9].

The mortality rate is also high because of the difficulty of the surgical act, the aggressivity of chondrosarcoma, as well as the high recurrence rate. Deloin recorded a mortality rate of $39 \%$ in one of his studies [19].

The purpose of surgical treatment is to completely remove the tumor, within oncological limits and to achieve a good functionality of the affected limb.

From a historical point of view, hemipelvectomy represented the elective surgical approach in order to remove the tumor within oncological limits, but this technique is currently reserved for complex and extreme cases, as most studies report that there is no significant statistical difference between hemipelvectomy and tumor resection followed by local anatomical reconstruction $[4,20,21]$. Multiple reconstruction methods are currently under development and continuously adapted to the type of resection performed and the instability secondary to the tumor removal [22].

According to Johnson and Satcher, the use of cement in pelvic reconstruction techniques showed very good results, as they have filled the remaining bone defect with cement, and then implanted a total hip prosthesis $[11,23]$.

\section{Conclusions}

Nowadays, the advancements in both surgical approaches and techniques allow the reconstruction of the joints and bone structures, with improved functional outcome, as opposed to the historical resolve that mostly involved amputation.

Bone defects repair using antibiotic impregnated cement drastically reduced the rate of postoperative infections, thus decreasing both morbidity and mortality.

In spite of technological advancement, long-term prognosis remains reserved in chondrosarcoma, duetoits specificaggressivity, resistance to chemo- and radiotherapy and high rate of recurrence.

In order to obtain a good outcome and an improved quality of life, the surgical treatment must be carefully planned and an increased attention must be paid to postoperative care and early detection of relapses.

\section{Acknowledgement}

All authors had equal contribution in writing this paper.

\section{References}

1. Mottard S, Sumathi VP, Jeys L. Chondrosarcomas, Orthopaedics and Trauma. 2010.

2. Santini-Araujo E, Kalil RK, Bertoni F, Park YK. Tumors and Tumor-Like Lesions of Bone For Surgical Pathologists. Orthopedic Surgeons and Radiologists. 2015, Springer-Verlag, London.

3. Campanacci MD. Bone and Soft Tissue Tumors - Clinical Features, Imaging, Pathology and Treatment. 1999, Springer, Vienna.

4. Pring ME, Weber KL, Unni KK, Sim FH. Chondrosarcoma of the pelvis. A review of sixty-four cases. J Bone Joint Surg Am. 2001; 83A:1630-42.

5. Lee FY, Mankin HJ, Fondren G et al. Chondrosarcoma of bone: an assessment of outcome. J Bone Joint Surg Am. 1999; 81:326-38.

6. Angelini A, Guerra G, Mavrogenis AF, Pala E, Picci P, Ruggieri P. Clinical outcome of central conventional chondrosarcoma. J Surg Oncol. 2012; 106:929-37.

7. Enneking WF, Dunham WK. Resection and reconstruction for primary neoplasms involving the innominate bone. J Bone Jt Surg Am. 1978; 60(6):731-46.

8. Kollender Y, Shabat S, Bickels J, Flusser G, Isakov J, Neuman Y et al. Internal hemipelvectomy for bone sarcomas in children and young adults: surgical considerations. Eur J Surg Oncol. 2000; 26(4):398-404. 
9. Puri A, Pruthi M, Gulia A. Outcomes after limb sparing resection in primary malignant pelvic tumors. Eur J Surg Oncol. 2014; 40:27-33.

10. Rodl RW, Hoffmann C, Gosheger G, Leidinger B, Jurgens H, Winkelmann W. Ewing's sarcoma of the pelvis: combined surgery and radiotherapy treatment. J Surg Oncol. 2003; 83:154-60.

11. Satcher RL Jr., O'Donnell RJ, Johnston JO. Reconstruction of the pelvis after resection of tumors about the acetabulum. Clin Orthop Relat Res. 2003; 409:209-217.

12. Guo W, Sun X, Ji T, Tang X. Outcome of surgical treatment of pelvic osteosarcoma. J Surg Oncol. 2012; 106:406410.

13. Asavamongkolkul A, Waikakul S. Using polypropylene mesh graft for soft tissue reconstruction in internal hemipelvectomy: a case report. World J Surg Oncol. 2012; 28(10):12410.

14. Zheng G, Jing L, Guo-Xian P, Xiang-Dong L, Zhen W. Pelvic reconstruction with a combined hemipelvic prostheses after resection of primary malignant tumor. Surgical Oncology. 2010; 19:95-105.

15. Buchholz HW, Gartmann HD. Infection prevention and surgical management of deep insidious infection in total endoprosthesis. Chirurg. 1972; 43:446-53.

16. Wahlig H, Dingeldein E, Buchholz HW, Buchholz M, Bachmann F. Pharmacokinetic study of gentamicin-loaded cement in total hip replacements. Comparative effects of varying dosage. J Bone Joint Surg Br. 1984; 66:175-9.

17. Donati D, Biscaglia R. The use of antibiotic-impregnated cement in infected reconstructions after resection for bone tumours. J Bone Joint Surg Br. 1998; 80:1045-50.

18. Mavrogenis AF, Soultanis K, Patapis P et al. Pelvic resections. Orthopedics. 2012; 35:e232-e243.

19. Welkerling H, Kratz S, Ewerbeck V, Delling G. A reproducible and simple grading system for classical chondrosarcomas. Analysis of 35 chondrosarcomas and 16 enchondromas with emphasis on recurrence rate and radiological and clinical data. Virchows Arch. 2003; 443:725e33.

20. Shin KH, Rougraff BT, Simon MA. Oncologic outcomes of primary bone sarcomas of the pelvis. Clin Orthop Relat Res. 1994; 304:207e17.

21. Deloin X, Dumaine V, Biau D, Karoubi M, Babinet A, Tomeno B, Anract P. Pelvic chondrosarcomas: Surgical treatment options. Orthopaedics \& Traumatology: Surgery \& Research. 2009; 95:393-401.

22. Salunkea AA, Shaha J, Warikooa V, Chakrabortya A, Sahijwania H, Sharmaa M, Jhaa R, Dhangea A, Pathakb S, Pandita J, Pruthic M, Pandyad S, Jaina A. Surgical management of pelvic bone sarcoma with internal hemipelvectomy: Oncologic and Functional outcomes. Journal of Clinical Orthopaedic and Trauma. 2017; 8:249-253.

23. Johnston JO, Gray RM. Hip reconstruction following internal hemipelvectomy for primary periacetabular sarcomas. Chir Organi Mov. 1990; 75:249-52. 